\title{
Interpretation of information that an instance is positive or negative in concept identification'
}

\author{
J. PETER DENNY and JOHN G. BENJAFIELD, ${ }^{2}$ Department of \\ Psychology, University of Western Ontario, London, Canada
}

Previous authors have speculated about the reasoning Ss have used to identify concepts more proficiently from positive than from negative instances. In the present study, a part of this reasoning, Ss' interpretation of information that an instance is positive or negative, was observed by means of novel measures. These were Ss' conclusions about the concept at each stage of the task, and $S s^{\prime}$ stimulated recall of the reasoning they used to draw each conclusion. Ss' made equally frequent but different misinterpretations of positive and negative instances. These misinterpretations appeared to be part of a particular reasoning strategy which yielded correct conclusions when applied to positive instances and incorrect conclusions when applied to negative instances.

It is well established that Ss identify concepts more proficiently from positive than from negative instances (Bourne, 1966; pp. 54-56). Although some speculations have been made about the strategies of reasoning which might underlie this phenomenon (Fryatt \& Tulving, 1963; Huttenlocher, 1962; Glanzer, Huttenlocher, \& Clarke, 1963), no observations of such strategies have been made. A part of these strategies which seemed worthy of initial investigation was S's interpretation of the information that an instance is positive or negative. In the present study, novel dependent variables were used to assess these interpretations so as to test the association between correctness of interpretation and concept identification proficiency.

Subjects and procedure. Fifteen undergraduate Ss were randomly assigned to either a positive instance or a negative instance task. The tasks shown in Table 1 utilized eight attributes which were letters, A through $H$. The letters were either present or absent in an instance, which was a row, e.g., AB DEFGH. The conjunctive concept contained four letters $A, D, E$, and $G$, all of which had to be present in a positive instance.

The positive task contained five positive instances, each marked + , the first containing all the attributes, and the others having one different attribute missing from among those not in the concept. The negative task contained the same first positive instance, followed by four negative instances, each marked -, and having one different attribute missing from among those in the concept. The instructions explained attributes, conjunctive concepts, positive and negative instances, but did not say how many attributes were in the concept. The task was presented to $S$ one instance at a time. Previously presented instances remained in view in the order of presentation.

Assessment of $\mathrm{Ss}^{\prime}$ Interpretations of Positive and Negative Instances. Two novel measures were used. First, Ss were required to state for each attribute, that they concluded it was in the concept (symbolized $\mathrm{I}$ ), not in the concept $(\mathrm{N})$, or that they did not know (?). They drew these conclusions for every attribute after the presentation of every instance and recorded them on a record sheet using the symbols $\mathrm{I}, \mathrm{N}$, and ?. Second, at the end of the task they were shown all their responses and all the instances,

Table 1

The Concept Identification Tasks

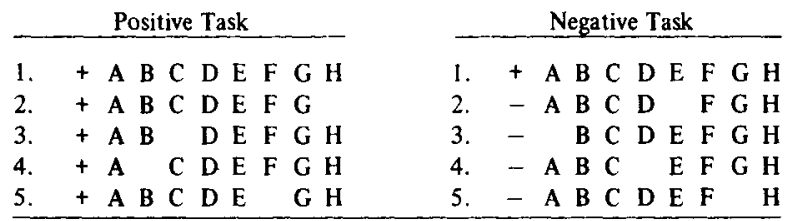

and interviewed about the reasoning they used to arrive at each $I$, $\mathrm{N}$, or ? response.

The Ss' interview responses were analyzed by objective procedures to determine the interpretation of the information that an instance was positive (made by positive task Ss), and the interpretation of information that an instance was negative (made by negative task $\mathrm{Ss}$ ). The scoring program (1) selected all statements containing "positive" or "negative" or their synonyms, (2) standardized the vocabulary of the statements by substituting standard terms for their synonyms, and (3) standardized the syntax of the statements so that all statements could be (4) compared to standard statements and thereby classified as to type of interpretation. The Ss' I, N, and ? responses were used to verify the interview analysis, by checking that a $S$ 's responses were logically consistent with his interpretation of positive or of negative instances. All responses were logically consistent with interpretations, although two Ss made additional responses purely as guesses, and three Ss sometimes responded ? because they were unsure of their interpretations.

The Ss' interpretations were further classified as correct or incorrect, depending upon whether or not they were logically consistent with the basic meanings of positive and negative instances given in the instructions.

Assessment of concept identification proficiency. Ss' concept identification proficiency was measured by the number of attributes which he correctly included in or excluded from the concept when asked for his conclusions at the end of the task. Since there were eight attributes, scores could range from 0 to 8 .

Results. Ss receiving the positive task drew significantly more correct conclusions that attributes were included in or excluded from the concept, $\overline{\mathrm{X}}=7.3$, than did $\mathrm{Ss}$ receiving the negative task, $\bar{X}=2.7, U=33, n_{1}=n_{2}=15, p<.002$. This confirms the findings of other studies using very similar concept identification tasks (e.g., Glanzer, Huttenlocher, \& Clarke, 1963).

Table 2 shows proficiency of concept identification as a function of correctness of interpretation and of positive or negative instances. To begin with, it can be seen that the number of Ss making correct and incorrect interpretations is the same for positive and negative instances. Analysis of variance and general partition of chi-square could not be applied to the proficiency scores due to small and unequal Ns, skewed distributions, and heterogeneity of variance. Consequently $U$ tests between all possible pairs of groups were performed which revealed that the Negative Task-Incorrect Interpretation group made significantly lower scores than each of the other three groups, and that the latter groups did not differ significantly among themselves. Thus, Ss receiving positive instances formed the concept proficiently whether they interpreted the instances correctly or not $(U=27$, $n_{1}=7, n_{2}=8$ ), whereas $S s$ receiving negative instances formed the concept proficiently if they interpreted the instances correctly, but not if they interpreted them incorrectly, $U=5, n_{1}=8, n_{2}=7$, $\mathrm{p}<.01$.

Of the eight Ss making incorrect interpretations in the positive task, seven gave the misinterpretation, "a positive instance means all the attributes present in the instance are in the concept,"

Table 2

Number of Ss Making Correct and Incorrect Interpretations and Median Number of Attributes Correctly Included in and Excluded from the Concept

\begin{tabular}{lccccccc} 
& \multicolumn{3}{c}{ Positive Task } & & \multicolumn{3}{c}{ Negative Task } \\
\cline { 2 - 4 } \cline { 6 - 8 } Interpretation & $\mathrm{f}$ & Median & Range & & $\mathrm{f}$ & Median & Range \\
\hline Correct & 7 & 8 & $6-8$ & 7 & 6 & $0-8$ \\
Incorrect & 8 & 8 & $4-8$ & & 8 & 0 & $0-2$ \\
\hline
\end{tabular}


whicas the correct interpretation was the reverse of this, " $a$ positive instance means all the atributes in the concept are present in the instance. "The eighth S's misinterpretation was, "a positive instance means all the attributes are present in the instance, because they are in the concept."

Of the eight Ss making incorrect interpretations in the negative task, four gave the misinterpretation, "a negative instance means that an attribute absent from the instance is not in the concept," and four gave, "a negatile instance indicates an attribute is absent from the instance." The correct interpretation was "a negative instance means that an attribute absent from the instance is in the concept."

Discussion. The results show that $\mathrm{Ss}$ misinterpreted positive and negative instances equally frequently, although the misinterpretations of each are different. More importantly, misinterpretations of negative instances were associated with poor concept identification proficiency whereas misinterpretations of positive instances were not. To understand why this was so, preliminary data on the other parts of Ss' strategies were examined. It appeared that misinterpretations of positive and negative instances were, for most Ss, a part of a strategy whose core was the reasoning that an attribute absent from an instance was not in the concept. This reasoning yields correct conclusions about the concept when applied to positive instances, but incorrect conclusions when applied to negative instances. Further studies of this strategy are underway.

\section{REFERENCES}

BOURNE, L. E. Human conceptual behavior. Boston: Allyn \& Bacon, 1966. FRYATT, M. J., \& TULVING, E. Interproblem transfer in identification of concepts involving positive and negative instances. Canadian Journal of Psychology, 1963, 17, 106-117.

GLANZER, M., HUTTENLOCHER, J., \& CLARKE, W. H. Systematic operations in solving concept problems. Psychological Monographs, 1963, 77, (Whole No. 564)

HUTTENLOCHER, J. Some effects of negative instances on the formation of simple concepts. Psychological Reports, 1962, 11, 35-42.

$$
\text { NOTES }
$$

1. Based on an MA thesis by the junior author supervised by the senior author and submitted to the Faculty of Graduate Studies, University of Western Ontario. This research was partially supported by National Research Council of Canada grants APA-81 and APA-137.

2. Now at Brandeis University. 